\title{
Various Approaches of Recognition of Digitally Modulated Signals
}

\author{
Bhawna \\ ECE Dept. Haryana College of \\ Tech. \& Management (Kaithal)
}

\author{
Mukhwinder Kaur \\ ECE Dept. Haryana College of \\ Tech. \& Management (Kaithal)
}

\author{
Dr.G.C.Lall \\ ECE Dept. Haryana College of \\ Tech. \& Management (Kaithal)
}

\begin{abstract}
Digital modulation techniques are use when the information signal is digital and the information signal is modulated by the amplitude, phase or frequency of a carrier. Various digital modulation techniques are used for various signal transmission. All these techniques provide versatility to the transmission medium and recognizing these modulation schemes is quite useful for the military and COMINT applications. All Digital modulation methods are based on some statistical parameters. Various recognition algorithms have been developed and still developing. The recognition algorithms are divided into two major groups maximum likelihood approach (MLA) and pattern recognition approach (PRA).Aim of this paper to describe different techniques of modulation recognition in brief along with various key features involve in these techniques, including all consideration of transmitter and receiver.
\end{abstract}

\section{Keywords}

ASK, PSK, SDR, COMINT, Modulation Recognition

\section{INTRODUCTION}

In digital communication system we transport digital data between two or more points. In radio communications usually it is achieved by adjusting a physical characteristic of a sinusoidal carrier, the frequency, phase, amplitude or a combination two. This is performed by the modulators and demodulators at the receiving end as real systems with a modulator at the transmitting end to impose the physical change to the carrier and a demodulator at the receiving end to detect the resultant modulation on reception.

Modulator and demodulator at receiving end as well as at transmitting points various communication signals are available with different types of modulation with different frequencies. It is obvious to identify and closely observe these signals for few applications. Some of the applications find Place for example signal confirmation and spectrum Management and other applications are also used for military purposes for example electronic warfare and threat analysis. For all electronic warfare techniques electronic support measures [1] [2] system play a important role as a means of information which is needed to conduct electronic counter measures (ECM), threat detection, Warning and target acquisition and homing. Under wireless communication due to the expanding of the in consumer electronics the demand of Parameters and on the selection of the important features applications, is resulted in comprehensively building the reliability and also provide the quality of service. This scenario has created a requirement to present intelligent addons the incoming signal. All Modulation types are supposed to be the signal signature in for communication Intelligence (COMINT) [3]
Firstly; when apply the signals to an improper demodulator it may be partially or totally damage the signal information of the data contents. Secondly when we have identified the modulation type, right demodulator can demodulate the signal to get back the information in the original form. Hence, modulation recognition is an important and crucial and functionalities in the wireless system, which has lead to the future technology of Cognitive Radio (CR) one of the most crucial tasks of a CR (Cognitive radio) Receiver is the total automatic recognition for the modulator step to recover or to get the signal that is exactly transmitted. All communication signals which are intercepted they have a great probability of uncertainty because of the inability to unidentified types of modulation as well as noise. Hence, in modulation classification algorithms have been generated and implemented. Feature extraction along with classification algorithm stages collect form important stage of a modulation recognition system. [1] Efficiency and effectiveness of the modulation recognition system almost all entirely depends on the initial knowledge of few of the signal stages and classification algorithm.

\subsection{Different modulation schemes}

Two types of modulations are mainly used Coherent and Incoherent Systems. FSK is main example we can generate coherently as well as non coherently the terms coherent and incoherent are frequently used for the generation and reception of digital modulation. When used to the process of modulation the term coherence it means the ability of the modulator to control the phase of the signal, not only the frequency.

For example Frequency Shift Keying (FSK) can be generated both coherently with an IQ modulator and incoherently with simply a Voltage Controlled Oscillator (VCO) and a digital voltage source, as shown in figure 1.

During the course of this paper, we have provided an basic overview of modulation Recognition approaches [8] which all are based on statistical properties for digital modulations including ASK2 (Amplitude Shift Keying2), ASK4, PSK2(Phase Shift Keying 2), PSK4, FSK2 (Frequency Shift

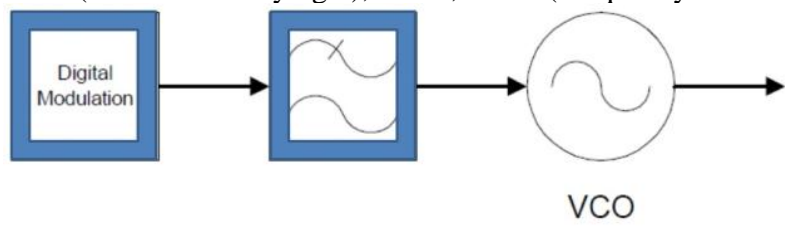

Fig.1 FSK Modulator using coherent modulation 
Keying 2), FSK4, QAM etc .again its application in field of COMNIT is discussed in last section of the paper.

\section{VARIOUS IMPORTANT EXTRACTION FEATURES}

The main characteristic and features which are used in case of classification are instantaneous amplitude, frequency, and phase and power spectral density of signal. [10]

The signal squared and the signal quadrupled is used to use out with the following key features of the interception of signal:

1) Envelop mean

2) Envelop variance

3) Location and magnitude of the two largest peaks in the signal spectrum

4) The spectral component magnitude being twice carrier frequency of squared signal

5) The spectral components magnitude being four times of carrier frequency of Quadrupled signal.

The different other statistical characteristics are developed from the signal's power spectral density, instantaneous amplitude, frequency and phase. Normally the classification of ASK2and PSK2 is not possible because in almost all the cases their constellations act as an important parameter. Various statistical parameters based on the mean, variance, and histograms have been used with AMC [6] [11]. All the statistical parameters can be calculated using the basic all key features. However many of the features need some basic of the signal parameters for example carrier frequency, pulse shape, time of arrival, initial phase, symbol rate, signal to Noise ratio, to be known or to be extracted.

\subsection{Need for modulation recognition}

Most of veiled and manifest operations of classification of modulation whether of analog or digital modulation technique

are performed because it is necessary to know the type of incoming signal. How to observe and identify modulated signals is necessary to know about valuable information [12] [13] [14]. The recognizers help to differentiate the signal in presence of (AWGN) along with presence of other signals the process of recognition is a most important intermediate step between the detecting and demodulating process.

\section{APPROACHES FOR MODULATION RECOGNITION}

Over the study of last 20 years a lot of modulation recognition techniques have been developed. Azzouz and Nandi made use of two vastly different Approaches [8] [11] [9] in order to differentiate modulated signals almost all the approaches can be divided into two major groups:

1) Maximum likelihood method

2) Pattern recognition method

These approaches are further explained in brief in the next section of the paper.

\subsection{Maximum likelihood method}

In maximum likelihood technique, the test statistics needs advance knowledge about the signal, although the decision rules are quite simple. The maximum likelihood approaches are able to provide an optimal solution by minimizing the possibility of false distribution if all other possibilities are met.

Within the maximum likelihood approach, AMC (Automatic modulation classification) is built using it as a multiple composite hypothesis-Testing problem, and also the histogram is applied to AMC. Hypothesis is solved with the help of different maximum likelihood techniques.

Various implementations of maximum AMC approaches are already been proposed for ML-AMC which is based on various possibilities regarding the unknown signal. In the maximum likelihood approach, types interpreted considering [10] it as a multiple hypothesis testing problem, where a hypothesis $\mathrm{H}$ is arbitrarily allotted to the $\mathrm{X}_{\mathrm{I}}$ modulation type of $\mathrm{m}$ different types. The ML classifier is based on the conditional probability density function. Different tests under different scenarios have been brought forward for AMR based different possibilities and conditions.

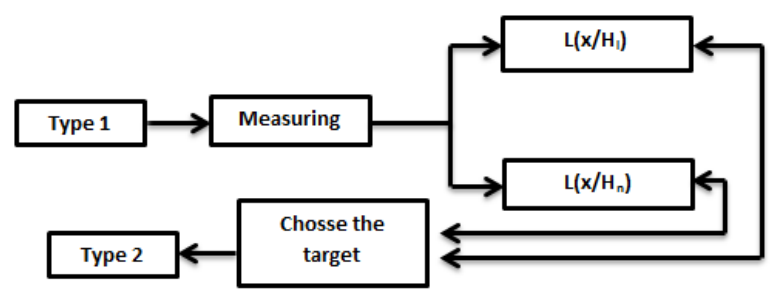

Fig.2 General Maximum Likelihood classifier

In special case the probability density functions of the above mentioned features are already known, [10] an average likelihood ratio test can be used to make available a absolute solution under modulation estimation.

Under average likelihood ratio test, the unknown parameters, for example a noisy signal symbol are considered as a random variable with $M I$ number of hypothetical probability density functions and also the logarithmic likelihood function for the it hypothesis is calculated.

If the true probability density functions meet with the hypothetical ones, the results are supposed to be maximized. [12] It can be written, the average likelihood ratio test will give improvised solutions which is based on the distributions of errors instead of the instantaneous errors. However, the average likelihood ratio test modulation classifier is not only computationally intensive but it also needs correct time, frequency, and phase estimations in order to convert the IF data to near the baseband.

When probability functions of the various features are not available, the generalized likelihood ratio test (GLRT) might be used which is considers all the available candidates as not known deterministic values. The generalized likelihood ratio test [8] is not an optimal estimator which is not the case in average likelihood ratio test but it has simple implementation. Generalized average ratio test might also be seen as a variance test with is having time-varying mean values. [12] Variance test is generally used modulation estimation in order to 
measure the feature fluctuations but it is pretty sensitive to the additive noise and channel fading.

The histogram test is also used for the linear Modulation classification [7] [8] this test makes use of very less power for Processing purpose. Variant features like phase, phase differences frequencies, distributions of amplitudes are plotted like histograms. Modulation scheme is finally recognized by statistically comparing the matching templates and histograms.

\subsection{Pattern recognition method}

In case of statistical pattern recognition approaches, complex decision rules are involved. General pattern recognition system consists of three parts: sensing, feature extraction and decision procedures.

The pattern vector presents features of a pattern or object. [15] The pattern vector might have useless information. Dimensionality of the pattern space should be decreased in order to simplify the design of the computational effort. In it decision procedure might contain decision functions, distance functions, or neural networks. These approaches provide good results for different values of SNR (Signal to noise ratio).The decision part requires artificial neural networks.

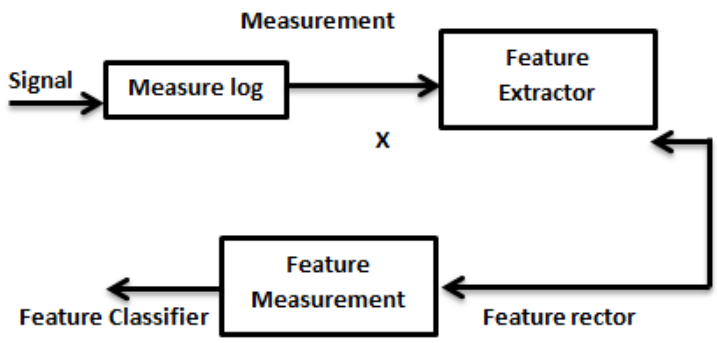

Fig.3 General Pattern recognition System

\subsection{Artificial Neural Network}

ANN is a well known mathematical model based on Biological neural network.ANN is good in finding the desired pattern of the data. IT also establishes the relationship between the input and output. They have been in use for many

Years for the purpose of modulation recognition [18] and even in present times also they are used with different genetic algorithms. The best quality of ANN is they can easily work in complex environment where other computations get fail. ANN is good in non linear mapping of the signal, as well as self adaptability ANN is employed for recognizing different M-ary modulations.

\subsection{ANN modulation recognizer for m-ary modulation techniques}

The modulation recognizer which is based on the ANN approach consists of the three main blocks

1) The block of preprocessing wherein the key input characteristics are extracted from each and every part of the signal frame.
2) The blocking order of training and learning to make way for classifier structure

3) The test block in order to make a decision about the modulation type of a signal.

The very first is used to differentiate in between the given modulation types apart from the decisions related to the estimation of the number of levels in the M- ary amplitude shift-keying (MASK) and the (MFSK ) frequency shiftkeying.

The differentiation between ASK2 and ASK4 is done through the use of another different network known as the second network, and also the differentiation between FSK2 and FSK4 is done through the use of a third network.[18]

From the first network, only two of the output decisionsnamely MASKS decision and MFSK decision -need the additional two networks to complete the differentiation. The second and third

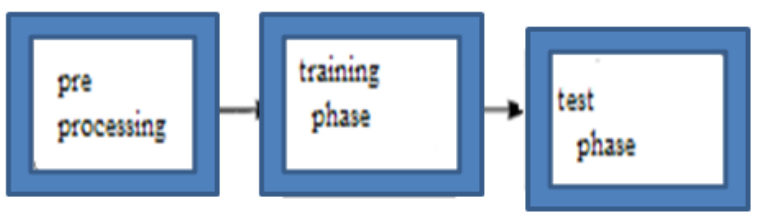

Fig.4 Functional blocks of ANN

Networks calculate the number of levels of the above mentioned two signals.

In case of the first network, it came to notice that the best ANN consists of three neuron layers. First hidden layer makes use of the log-sigmoid as the activation function; [1] [6] linear function is used as the activation function in case of the second hidden layer and the third layer makes use of the logsigmoid function as the activation function.

In the case of second and third networks, only one activation function (log-sigmoid) is used making it very simple. But in case of excess of unnecessary information their performance gets poor in terms of convergence so other algorithms or genetic algorithms are used along with this that increases the speed and recognizing ability.

\section{APPLICATIONS FOR COMINT}

Historically, COMINT (Communication Intelligence) systems have dependent on the manual modulation recognition of measured parameters in order to provide classification of different emitters. But, recently automatic modulation recognition systems have come into picture. [12]

One of the historic models of modulation recognizers makes use of a bank of demodulators; each one of them is designed for single type of modulation. By observing the demodulator Outputs one can decide the modulation type of the received signal. [13] [16] this requires highly skilled operators as well as long signal durations. The Automation of this kind of recognizer is performed by using a set of intelligent decision algorithms at the demodulator outputs. But, the implementation using in this scenario is complicated and 
needs excessive computer storage. The number demodulators used limit the modulation types that can be recognized.

Automatic modulation recognition seems to be more effective than manual modulation recognition because it can integrate the automatic modulation recognizer with an electronic support measurement [19] [20] receiver which would allow an operator to enhance his efficiency and his ability to analyze the various activities in the frequency band of interest. Hence, in advanced ESM systems, sophisticated electronic machines are used instead of an operator.

The main aim of any surveillance system is supposed to be threat recognition by comparing the features of the intercepted emitters with a catalogue of reference characteristics.

\section{APPLICATIONS FOR SDR}

The new generation of communication is SDR which is very useful for the rise of $4 \mathrm{G}$ so that systems are quite flexible offering interoperability as well as even overcome the operation cost. These systems convert hardware functions to the software realization.SDR receiver shown in fig. 5 do not require any permissible change in the Integrated circuit for different digitally modulated signals. A RF front end is basically employed in a particular SDR system.

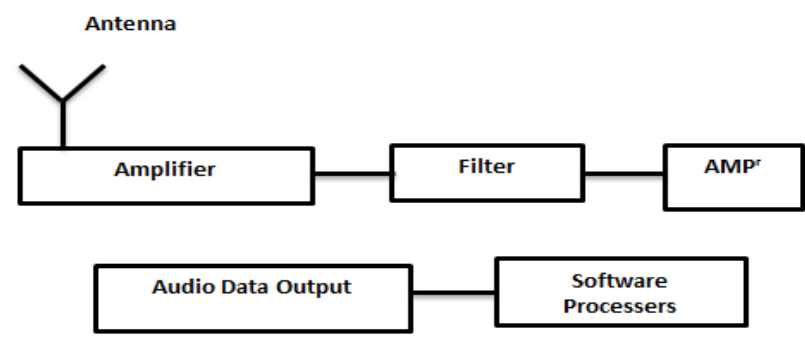

Fig.5 Block diagram of SDR reciever

Antenna signal is filtered, amplified according to the need and finally converted from analog to digital signal. For digital signal processing proper software is employed performing different functions like of converting pass band to the

baseband and demodulating the signal. DSP or general purpose processor is taken in use for processing the software. Lesser bit rate requirement of the $4 \mathrm{G}$ systems is fulfilled by

maximizing the channel capacity with the use of dynamic Channel assignment scheme.

\section{CONCLUSION}

The basic aim of the paper is to explain about the different automatic modulations recognition algorithms along with a number of important key features of extraction proposed for these recognizers.

It has been found that when using maximum likelihood approach, it depends on mean, variance and used signal parameter pulse shape, time of arrival initial phase and requires more signal parameters knowledge as compare to pattern recognition approach which is better result base than that of previous study and on simulation give better results While pattern recognition, it provides an optimal solution for different SNR values using artificial neural network.
Application of recognition approach in the field of COMINT is also discussed in brief; these all application can be used for future study to give practical results on simulation. These all comparison based on previous study of review and on base of new review better results can be obtained, using SDR overcome the operation cost which is main attractive feature.

\section{ACKNOWLEDGMENTS}

Authors of the paper are thankful to anonymous authors and research contributor for their valuable efforts and findings.

\section{REFERENCES}

[1] E.E Azzouz and A.K. Nandi, "Automatic Modulation Recognition of Communication Signals", Kluwar Academic Publishers, 1996.

[2] D. Linda Essentials of cognitive radio, Cambridge Wireless Essentials Series, Cambridge University Press, 2009.

[3] O.A. Dobre and Y. Bar-Ness Blind Modulation Classification: A Concept Who's Time has Come IEEE/Sarnoff Symposium, pp. 223U" 228 April 18U" 19, 2005.

[4] D. L. Guen, A. Man sour, "Automatic Recognition Algorithm for Digitally Modulated Signals", International Conference on Signal Processing, Pattern Recognition, and Applications Crete, Greece, 25-28 June,2002.

[5] K .N. Haq, A. Mansur, Sven Nordholm, "Comparison of digital modulation classification based on statistical approach", 10thPostgraduate Electrical and Computer Symposium Perth Australia, September 2009.

[6] S.S. Soliman and Z.S. Hsue, "Signal classification using statistical moments,"IEEE Transactions on Communications, vol. 40(5), pp. 908-916, May 1992

[7] Z.S. Hsue and S.S. Soliman, "Automatic modulation classification using zero-crossing IEEE Proc. Part F,

Radar and signal processing, vol. 137 (6), pp. 459-464, December 1990.

[8] G.Acosta, "OFDM simulation using Mat Lab", Report, Smart Antenna Research Laboratory Georgia Institute of Technology, Georgia, USA, August 2000.

[9] Li Tieying, Cui yan,"A design of neural classifier based on rough sets" [J]. Computer Engineering and Applications, 2005, 32.

[10] Adel Metref, Daniel Le Guennec, Jacques Palicot "A new digital modulation recognition technique using the phase detector reliability " 2010 .

[11] HU You-qiang, LIU Juan, TAN Xiao-hang "Digital modulation recognition based on instantaneous information "June 2010.

[12] Fatima K. Faek," Digital Modulation Classification Using Wavelet Transform and Artificial Neural network" (JZS) Journal of Zankoy Suleiman 2010.

[13] Asoke K. Nandi, E. E. Azzouz "Algorithms for Automatic Modulation Recognition of Communication Signals" IEEE Transactions On Communications, Vol. 46, No. 4, April 1988. 
[14] Liang Hong K.C. Ho" Identification of Digital Modulation Types Using the Wavelet Transform", vol2, pp. 20.2.1-20.2.6, October 2010.

[15] Mobien shoaib, Alharbi Harza, Alturki Fahd "Robustness of digital modulated signals against variation in $\mathrm{Hf}$ noise model", EURASIP journal on wireless communication network, 2011.

[16] Wu Min.The research of Rough Set attribute reduction algorithm in numeral character recognition [D].Hefei University of Technology Master Dissertation, 2009.

[17] N Ahmadi, “, Modulation classification based on constellation using TTSAS approach", Journal of recognition research, May 2010.
[18] Zhao F., Hu Y.and SH.,Hao, 'Classification using wavelet packet decomposition and support vector machine for digital modulation', Journal of system Engineering and Electronics, August 2009,19,914-918.

[19] Khandker Nada Haq, Ali Mansour, Sven Nordholm" Recognition of Digital Modulated Signals based on Statistical Parameters ", 4th IEEE International Conference on Digital Ecosystems and Technologies (IEEE DEST 2010).

[20] Z.S. Hsue and S.S. Soliman, "Automatic modulation classification using zero-crossing IEEE Proc. Part F, Radar and signal processing, vol. 137 (6), pp. 459-464, December 1990. 